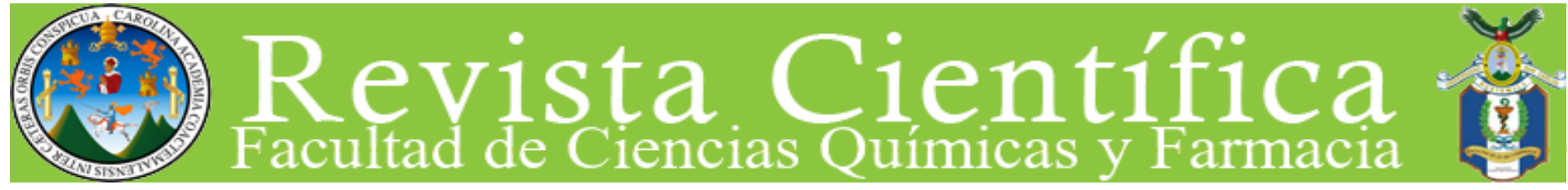

\title{
Frecuencia de síndrome metabólico en hombres y mujeres de 45-65 años que asisten al laboratorio clínico del Hospital Nacional de San Marcos
}

\author{
Frequency of metabolic syndrome in men and women aged \\ 45-65 years who attend the clinical laboratory of the National \\ Hospital of San Marcos
}

\author{
Saquic, Marta Julia; Velásquez Velásquez, Deris Roselvy; Valdés de \\ García, Alba Marina
}

\author{
Marta Julia Saquic \\ smarta290@gmail.com \\ Departamento de Bioquímica, Escuela de Química \\ Biológica, Facultad de Ciencias Químicas y Farmacia, \\ Universidad de San Carlos de Guatemala, Guatemala \\ Deris Roselvy Velásquez Velásquez \\ velasquezdros@gmail.com \\ Departamento de Bioquímica, Escuela de Química \\ Biológica, Facultad de Ciencias Químicas y Farmacia, \\ Universidad de San Carlos de Guatemala, Guatemala \\ Alba Marina Valdés de García \\ albavaldesdegarcia@gmail.com \\ Departamento de Bioquímica, Escuela de Química \\ Biológica, Facultad de Ciencias Químicas y Farmacia, \\ Universidad de San Carlos de Guatemala, Guatemala
}

\section{Revista Científica}

Facultad de Ciencias Químicas y Farmacia,

Universidad de San Carlos de Guatemala, Guatemala

ISSN-e: 2224-5545

Periodicidad: Semestral

vol. 30, núm. 1, 2021

almadariaga1@gmail.com

Recepción: 29 Abril 2021

Aprobación: 19 Agosto 2021

DOI: https://doi.org/10.54495/Rev.Cientifica.v30i1.15

URL: http://portal.amelica.org/ameli/jatsRepo/50/502283011/ index.html

Licencia: CC-BY 4.0
Resumen: El síndrome metabólico (SM) es un conjunto de alteraciones metabólicas e inflamatorias presentes en forma simultánea o secuencial, aumentando el riesgo de las enfermedades cardiovasculares (ECV) y diabetes mellitus tipo II (DM2). El objetivo de este estudio analítico fue comparar la frecuencia de SM y la triada más frecuente de sus componentes, en mujeres y hombres de 45 a 65 años que asistieron al laboratorio clínico del Hospital Nacional de San Marcos de enero a marzo del año 2020; utilizando los criterios del Programa Nacional de Educación sobre el Colesterol y el Panel III de Tratamiento del Adulto (NCEP-ATP III). El muestreo fue no probabilístico con 300 participantes divididos por afijación simple en 150 hombres y 150 mujeres; los datos bioquímicos, antropométricos y conductuales se analizaron en Epi Info versión 7.2.2.6. La frecuencia global de SM fue de 65.0\%, en mujeres $76.7 \%$ y $53.3 \%$ en hombres; la comparación de la frecuencia de SM entre hombres y mujeres fue significativa $(p<.001)$. Tener familiares con DM2 fue significativo en los hombres con SM. La tríada más frecuente en el 73.0 $\%$ de mujeres y en el $51.2 \%$ de hombres fue niveles de colesterol HDL disminuido, hipertrigliceridemia; difiriendo como tercer componente circunferencia abdominal aumentada e hiperglucemia respectivamente.

Palabras clave: Síndrome metabólico, enfermedades cardiovasculares, diabetes mellitus.

\begin{abstract}
The metabolic syndrome (MS) is a set of metabolic and inflammatory alterations they appear simultaneously or sequentially, increasing the risk of cardiovascular disease (CVD) and type II diabetes mellitus (DM2). The objective of this analytical study was to compare the frequency of MS in women and men aged 45 to 65 years old who attended the clinical laboratory of the National Hospital of San Marcos from January to March 2020; using the criteria of the National Cholesterol Education Program and Adult Treatment Panel III (NCEP-ATP III). The sampling was non-probabilistic with 300 participants divided by simple affixation into 150 men and 150 women; biochemical, anthropometric, and behavioral data were analyzed in Epi Info version 7.2.2.6.
\end{abstract}




\begin{abstract}
The global frequency of MS was $65.0 \%$, in women 76.7 $\%$ and $53.3 \%$ in men; the comparison of the frequency of MS between men and women was significant $(p<.001)$. Having relatives with DM2 was significant in men with MS. The most frequent triad in $73.0 \%$ of women and in $51.2 \%$ of men was decreased HDL cholesterol levels, hypertriglyceridemia; differing as a third component increased abdominal circumference and hyperglycemia respectively.
\end{abstract}

Keywords: Metabolic syndrome, cardiovascular diseases, diabetes mellitus, hypertriglyceridemia and hyperglycemia.

\section{INTRODUCCIÓN}

El SM en el siglo XXI es un problema de salud pública mundial que ocasiona alta morbilidad y mortalidad por sus complicaciones, debido al riesgo para el desarrollo de DM2 y de ECV que son las principales causas de muerte en todos los países de Latinoamérica con el subsecuente incremento en el costo para la atención de estos pacientes y el impacto sobre su calidad de vida (Lahsen, 2014).

El SM se define por un conjunto de factores bioquímicos, fisiológicos y metabólicos interconectados que aumentan en forma directa el riesgo de desarrollar enfermedades crónicas como ECV, accidente cerebrovascular, DM2 y cáncer (Kee et al., 2017; McKenney \& Short, 2011).

Entre los factores que constituyen el síndrome están la resistencia a la insulina (RI), adiposidad visceral, dislipidemia aterogénica, disfunción endotelial, susceptibilidad genética, hipertensión arterial (HTA), estado de hipercoagulabilidad y el estrés crónico. Se conoce que la obesidad visceral y la RI se caracterizan por la producción de adipocitocinas anormales, como el factor alfa de necrosis tumoral, interleucina-1(IL-1) IL-6, leptina y adiponectina (Grundy et al., 2005).

La interacción entre los componentes del fenotipo clínico del síndrome con su fenotipo biológico (RI, dislipidemia) contribuye al desarrollo de un estado proinflamatorio y a una inflamación vascular subclínica crónica que da como resultado los procesos ateroescleróticos (Wilson, D’Agostino, Parise, Sullivan, \& Meigs, 2005).

SM confiere un aumento de cinco veces en el riesgo de DM2 y dos veces el riesgo de desarrollar ECV durante los próximos 5 a 10 años (Alberti et al., 2009). Adicionalmente, al comparar las personas sin SM con las que lo tienen, se observa que los accidentes cerebrovasculares aumentan de dos a cuatro veces, los infartos al miocardio (IM) de tres a cuatro veces y la muerte por dicho evento se duplica. (Alberti \& Zimmet, 2005) independientemente de una historia previa de eventos cardiovasculares (Olijhoek et al., 2004).

Se considera el SM como un factor de riesgo de primer orden para la aparición de complicaciones aterotrombóticas. Por lo tanto, su presencia o ausencia debe considerarse un indicador de riesgo a largo plazo. Por otro lado, el corto plazo (5 a 10 años). El riesgo a corto plazo se calcula mejor utilizando los algoritmos clásicos (Framingham, Registre Gironí del Cor [REGICOR]) ya que incluyen edad, género, colesterol LDL y tabaquismo (Grundy et al., 2005).

Las personas con SM, en etapas tempranas tienen cambios deletéreos en cada uno de los órganos blanco que algunas veces son subclínicas y posiblemente reversibles (Huang, 2009; Tsukiyama et al., 2016). A consecuencia de la presencia de comorbilidades del SM la Organización Mundial de la Salud (OMS) le ha dado Código de la Clasificación Internacional de Enfermedades (CIE-9) (277.7).

Los estudios realizados en América Latina han revelado que la prevalencia de SM en adultos oscila entre $25.0 \%$ a $45.0 \%$; correspondiendo el $34.0 \%$ a la población urbana y el $32.5 \%$ a la rural (Cruz, Serrano, Jiménez, Villalobos, \& Murguía, 2015; López et al., 2013). Otros estudios realizados en poblaciones latinoamericanas de Chile, Colombia, México, Perú y Venezuela revelaron una alta prevalencia de SM que 
varió de $14.0 \%$ a $31.6 \%$ dependiendo de los criterios para el diagnóstico clínico y las características de la población de estudio (Ninatanta, Núnez, García, \& Romani, 2016).

La iniciativa centroamericana de diabetes (CADMI) en el periodo 2003 al 2006, realizó un estudio para determinar la frecuencia de SM. Los resultados indicaron que hubo una mayor proporción de personas con SM en el grupo de 20 a 39 años y menor en el grupo de adulto mayor. La prevalencia global en Centroamérica fue $30.3 \%$, siendo muy similar a la reportada en países desarrollados. Se evidenció como triada de factores de riesgo la obesidad central, hipertrigliceridemia y niveles bajos de C-HDL, condición que demostró heterogeneidad entre los países. (Wong, 2012).

El estudio de Evaluación múltiple de factores de riesgo cardiovascular en América Latina (CARMELA) investigó la frecuencia de SM usando los criterios del NCEP-ATP III, en siete ciudades de América Latina, informando una frecuencia general del $21.0 \%$ de SM. En general el SM fue más frecuente en mujeres que en hombres, $22.0 \%$ y $20.0 \%$. La frecuencia del SM aumentó con la edad, notablemente en mujeres (Escobedo et al., 2009).

En Guatemala, las enfermedades crónicas no transmisibles (ECNT) durante los últimos años han aumentado la morbilidad, registrándose un $15.0 \%$ de HTA y $17.0 \%$ de DM2 y una mortalidad de $29.0 \%$ por DM2, estas enfermedades afectan en su mayoría a la población a partir de los 40 años en hombres y mujeres (Ministerio de Salud Pública y Asistencia Social [MSPAS], 2018). Tzorin y colaboradores (2015), en un estudio reportaron la frecuencia global de SM de $13.8 \%$, en trabajadores del campus central de la Universidad de San Carlos de Guatemala. En el 2016, el estudio realizado en Santa Catarina Ixtaguacán del departamento de Sololá, se determinó la prevalencia de SM en mujeres indígenas mayores de 45 años, obteniéndose un 35.0 $\%$, utilizando los criterios del NCEPT-ATP III (Villalta, 2016).

En el departamento de San Marcos, área Suroccidental se desconoce la prevalencia de SM, lo que no permite conocer el riesgo de ECV y DM2, los factores de riesgo asociados y su relación con SM. El objetivo de este estudio analítico fue establecer la frecuencia de SM en la población estudiada, mediante el criterio del NCEPT-ATP III, para diagnosticar la presencia de SM y determinar los principales factores de riesgo que lo promueven (Bolado, Rivera, Soto, Blasco, \& Romeero, 2015; NCEP, 2001).

\section{Materiales Y MÉTODOS}

El estudio es analítico realizado en San Marcos de enero a marzo de 2020, con un muestreo no probabilístico de 300 participantes comprendido entre 45 a 65 años, divididos por afijación simple en 150 hombres y 150 mujeres distribuidos en 4 grupos etarios: de 45 a 50, 51 a 55, 56 a 60 y 61 a 65 años y que asistieron a la consulta externa del Hospital Nacional de San Marcos.

En el estudio se incluyeron a los participantes que completaron la boleta con la información demográfica que incluía la edad, sexo, medidas antropométricas, antecedentes familiares de diabetes mellitus, enfermedades cardiovasculares, cerebrovasculares. Además, se evaluó algunos hábitos, que de acuerdo con la literatura son factores predisponentes como el tabaquismo, ingesta de alcohol, e inactividad física. Al relacionar la información colectada con los resultados de laboratorio clínico se evaluó si la persona tenía factores predisponentes para el desarrollo del síndrome metabólico. Además, que cumplieron los criterios de inclusión (residir en el departamento de San Marcos, ayuno de 14 h, orden médica para determinación de los analitos bioquímicos).

Se contó con un consentimiento informado previo a la participación en el estudio, se realizaron las mediciones antropométricas como diámetro de la cintura, que se determinó rodeando el abdomen con cinta métrica anotando centímetros. Se realizó la determinación de peso y talla en posición de pie, descalzo, utilizando la balanza calibrada. Con la determinación de la presión arterial se solicitó al participante que se sentara en una posición adecuada y tomó con esfingomanómetro. Se tomaron muestras sanguíneas para las determinaciones de glucemia en ayunas, colesterol total, triglicéridos y colesterol de alta densidad (C- 
HDL). Toda la metodología bioquímica fue estandarizada según los procedimientos de VITROS 250. El programa de control de calidad interno para cada analito se realizó de forma intracorrida, y extracorrida con sueros control VITROS ${ }^{\bullet}$. Los resultados obtenidos estaban dentro dos desviaciones estándar del control y con un coeficiente de variación menor del 10.0 \%. La presencia de SM en los participantes se determinó de acuerdo a los criterios del Programa nacional de educación en colesterol-III panel de tratamiento adultos (NCEP-ATPIII-2005), el cual presenta la combinación de tres de los siguientes componentes: circunferencia de cintura $\geq 102 \mathrm{~cm}$ en hombres $\mathrm{y} \geq 88 \mathrm{~cm}$ en mujeres; niveles séricos de triglicéridos $\geq 150 \mathrm{mg} / \mathrm{dL}$; niveles séricos de colesterol HDL $<40 \mathrm{mg} / \mathrm{dL}$ en hombres y $<50 \mathrm{mg} / \mathrm{dL}$ en mujeres; presión arterial $\geq 130 / 85$ $\mathrm{mmHg}$; glucemia en ayunas; $\geq 110 \mathrm{mg} / \mathrm{dL}$ o existencia de diabetes señalada por el participante. El sobrepeso y obesidad fueron definidos como índice de masa corporal (IMC) $\geq 24.5$ y $30 \mathrm{~kg} / \mathrm{m} 2$, respectivamente (NCEP, 2001).

Se utilizó el programa Microsoft Excel 2013 para crear una base de datos, con los resultados (datos de análisis bioquímicos e información de boleta). Con el software Epi Info versión 7.2.2.6 se procedió a analizarlos de acuerdo con los objetivos, los datos se presentaron como porcentajes, intervalos de confianza. Se determinó la frecuencia de SM y la asociación con los posibles factores de riesgo. La determinación de la combinación más frecuente de las alteraciones metabólicas en los participantes se realizó mediante tablas de contingencia (variables cruzadas con dos resultados), prueba de Ji2 de asociación y cálculo de Prevalence Odds Ratio (POR) para evaluar asociación con factores de riesgo y $S M$. Un valor de POR $<1.0$, establece que hay asociación negativa entre el factor de riesgo y la enfermedad o que puede ser un factor protector; por otro lado, si el valor de POR = 1 , indica que no existe asociación, y si el POR $>1$, indica que el factor de riesgo está asociado con la enfermedad, para estimar la precisión de POR se utiliza un intervalo de confianza del $95 \%$. Finalmente, para estimar en los participantes el riesgo de padecer un evento cardiovascular en los próximos 10 años,se utilizó la calculadora para riesgo cardiovascular de Hipocampo.org (https://hipocampo.org/rv/ riesgo-cardiovascular.asp) cálculo basado en el Framingham Heart Study.

\section{Resultados}

$\mathrm{Al}$ analizar los datos sociodemográficos, en este estudio, se encontró que el rango 45 a 50 años obtuvo mayor número de participantes hombres $34.7 \%$ (52/150) y mujeres $27.3 \%$ (41/150); las ocupaciones predominantes fueron agricultor $40.0 \%(60 / 150)$ y ama de casa $76.0 \%(114 / 150)$ (Tabla 1$)$. 
TABLA 1.

Frecuencia de datos sociodemográficos en participantes que asistieron al Hospital Nacional de San Marcos.

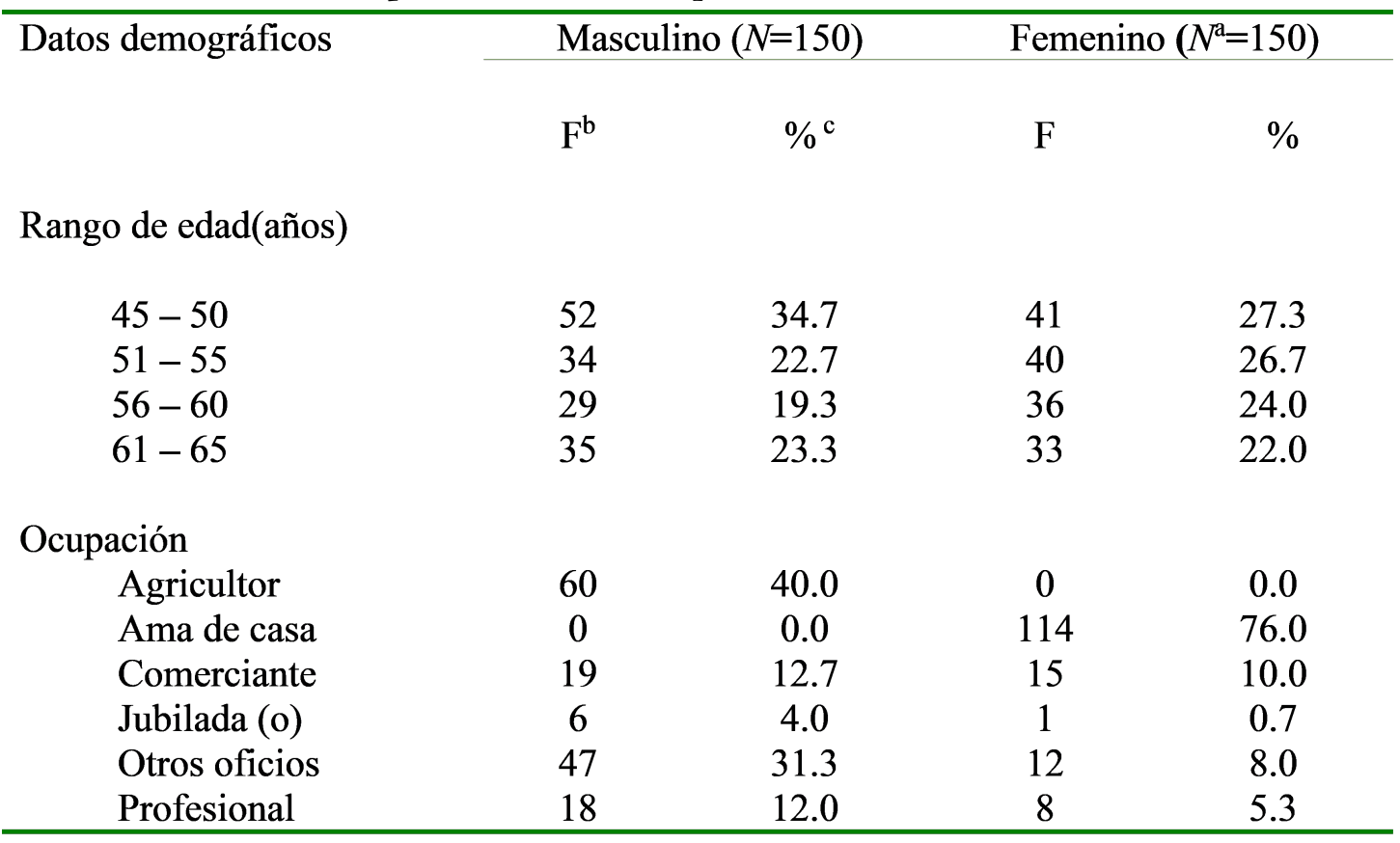

Nota: Na: número de muestra; Fc: frecuencia; \%d: porcentaje

La frecuencia global de SM fue de $65.0 \%$, (Tabla 2) con una mayor frecuencia de mujeres $76.7 \%(115 / 150)$ que de hombres con $53.3 \%(80 / 150)$, el $\mathrm{Chi}^{2}(17.8)$ indica que existe diferencia significativa en la frecuencia de SM entre hombres y mujeres $(p<.001)$.

TABLA 2.

Frecuencia de síndrome metabólico en participantes que asisten al Hospital Nacional de San Marcos.

\begin{tabular}{|c|c|c|c|c|c|c|c|c|c|c|}
\hline \multirow[t]{2}{*}{$\begin{array}{l}\text { Síndrome } \\
\text { metabólico }\end{array}$} & \multicolumn{2}{|c|}{$\begin{array}{l}\text { Masculino } \\
(n=150)\end{array}$} & \multicolumn{2}{|c|}{$\begin{array}{c}\text { Femenino }\left(n^{b}\right. \\
=150)\end{array}$} & \multirow{2}{*}{$\begin{array}{c}\mathrm{POR}^{\mathrm{h}} \\
0.3478\end{array}$} & \multirow{2}{*}{$\begin{array}{l}\mathrm{IC}^{\mathrm{e}} 95 \% \\
{[0.2,0.5]}\end{array}$} & \multirow{2}{*}{$\begin{array}{c}\mathrm{Chi}^{2 \mathrm{~g}} \mathrm{y} \\
\text { Valor } \\
p^{f} \\
17.8\end{array}$} & \multicolumn{2}{|c|}{$\begin{array}{c}\text { Frecuencia } \\
\text { global }(N=300)\end{array}$} & $\mathrm{IC}^{\mathrm{e}} 95 \%$ \\
\hline & $\mathrm{F}^{\mathrm{c}}$ & $\%^{d}$ & $\mathrm{~F}$ & $\%$ & & & & $\mathrm{~F}$ & $\%$ & {$[29.6,40.6]$} \\
\hline $\mathrm{Si}$ & 80 & 53.3 & 115 & 76.7 & & & $<.001$ & 195 & 65.0 & \\
\hline No & 70 & 46.7 & 35 & 23.3 & & & & 105 & 35.0 & {$[59.3,70.3]$} \\
\hline Total & 150 & $\begin{array}{c}100 . \\
0\end{array}$ & 150 & $\begin{array}{c}100 . \\
0\end{array}$ & & & & 300 & 100.0 & \\
\hline
\end{tabular}

\footnotetext{
Nota: Na: número de muestra; nb: número de participantes; Fc: frecuencia; \%d: porcentaje; ICe $95 \%$ : intervalo de confianza al 95\%; Valor pf: nivel significancia ( $\mathrm{p}<.05)$; Chi2g: estadístico chi cuadrado; PORh: Prevalence Odds Ratio
}

En la evaluación de las enfermedades crónicas asociadas y síndrome metabólico, se encontró que en los hombres existe asociación con SM y tener familiares con DM2 $(p<.0216)$. (Tabla 3 )

$\mathrm{Y}$ con respecto a la asociación de $\mathrm{SM}$ en los diferentes grupos etarios, en que se dividió la muestra, se encontró que en hombres la edad está asociada en la década de los 40 y 60 años, $\left(\mathrm{chi}^{2}=9.26, p<.010\right)$. En tanto que, en las mujeres, la edad no está asociada específicamente a cada una de las décadas, la frecuencia de SM es similar en todas las décadas estudiadas $\left(\mathrm{chi}^{2}=0.4991, p=.779\right)$. 
TABLA 3.

Frecuencia de enfermedades crónicas asociadas y síndrome metabólico

\begin{tabular}{|c|c|c|c|c|c|c|c|c|c|c|c|c|c|c|}
\hline \multirow{3}{*}{ Características } & \multicolumn{7}{|c|}{ Masculino $\left(N^{\mathrm{a}}=150\right)$} & \multicolumn{7}{|c|}{ Femenino $(N=150)$} \\
\hline & \multicolumn{2}{|c|}{$\begin{array}{c}\text { Con } \mathrm{SM}^{\mathrm{b}} \\
n^{\mathrm{c}}=80\end{array}$} & \multicolumn{2}{|c|}{$\begin{array}{c}\text { Sin SM } \\
n=70\end{array}$} & \multirow[t]{2}{*}{$\mathrm{POR}^{\mathrm{f}}$} & \multirow[t]{2}{*}{$\begin{array}{c}\mathrm{IC}^{\mathrm{g}} 95 \\
\%\end{array}$} & \multirow[t]{2}{*}{$\begin{array}{c}\text { Valor } \\
p^{\text {h }}\end{array}$} & \multicolumn{2}{|c|}{$\begin{array}{c}\text { Con SM } \\
n=115\end{array}$} & \multicolumn{2}{|c|}{$\begin{array}{c}\text { Sin SM } \\
n=35\end{array}$} & \multirow[t]{2}{*}{ POR } & \multirow[t]{2}{*}{ IC $95 \%$} & \multirow[t]{2}{*}{$\begin{array}{c}\text { Valor } \\
p\end{array}$} \\
\hline & $\mathrm{F}^{\mathrm{d}}$ & $\%$ e & $\mathrm{F}$ & $\%$ & & & & $\mathrm{~F}$ & $\%$ & $\mathrm{~F}$ & $\%$ & & & \\
\hline \multicolumn{15}{|c|}{ Familiar consanguíneo con diabetes mellitus tipo 2} \\
\hline $\mathrm{Si}$ & 40 & 64.5 & 22 & 35.5 & 2.2 & {$[1.1,4.3]$} & .0216 & 57 & 78.1 & 16 & 21.9 & 1.2 & {$[0.5,2.5]$} & .6908 \\
\hline No & 40 & 45.5 & 48 & 54.5 & & & & 58 & 75.3 & 19 & 24.7 & & & \\
\hline \multicolumn{15}{|c|}{ Familiar consanguíneo con enfermedad cardiovascular } \\
\hline $\mathrm{Si}$ & 19 & 47.5 & 21 & 52.5 & 0.7 & {$[0.3,1.5]$} & .3894 & 44 & 71.0 & 18 & 29.0 & 0.5 & {$[0.2,1.3]$} & .1674 \\
\hline No & 61 & 55.4 & 49 & 44.6 & & & & 71 & 80.7 & 17 & 19.3 & & & \\
\hline
\end{tabular}

Nota: Na: número de muestra; SMb: Síndrome metabólico; nc: número de participantes; Fd: frecuencia; \%e: porcentaje; PORf: Prevalence Odds Ratio; ICg $95 \%$ : intervalo de confianza al 95\%; Valor ph: nivel significancia ( $<$ < .05).

$\mathrm{Al}$ analizar las combinaciones de los componentes del SM que presentan los participantes, establecidos de acuerdo con criterios del NCEP-ATP II, se observa en la Figura 1, que la tríada más frecuente, en el 73.0 \% de participantes femeninas fue la circunferencia abdominal aumentada, nivel sérico de C-HDL disminuido e hipertrigliceridemia. En el $51.2 \%$ de los participantes masculinos la tríada más frecuente fue hiperglucemia en ayunas, nivel sérico disminuido de C-HDL e hipertrigliceridemia.

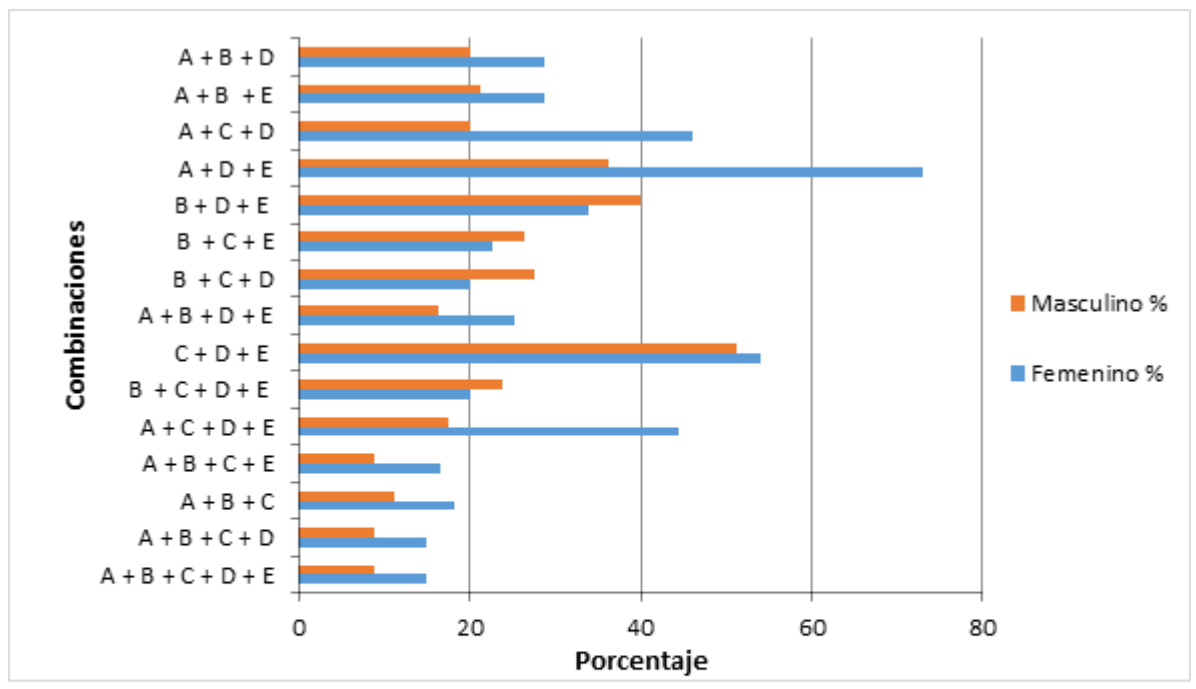

Figura 1. Combinación de los componentes de síndrome metabólico según el NCEP-ATP III encontrados en los participantes del Hospital Nacional de San Marcos. Nota: A: Circunferencia abdominal aumentada (femenino $\geq 88 \mathrm{~cm}$ y masculino $\geq 102 \mathrm{~cm}$ ); B: Presión arterial ( $\geq 130 / 85 \mathrm{mmHg}$ en ambos géneros); C: Glucosa en ayunas aumentada ( $\geq 110 \mathrm{mg} / \mathrm{dL}$ en ambos géneros); $\mathrm{D}$ : nivel sérico de C-HDL disminuidos $(\leq 50 \mathrm{mg} / \mathrm{dL}$ en femenino $\mathrm{y} \leq 40 \mathrm{mg} / \mathrm{dL}$ en masculino) y E: nivel sérico de triglicéridos aumentados ( $\geq 150 \mathrm{mg} / \mathrm{dL}$ en ambos géneros). 


\section{Discusión}

La frecuencia global de SM fue de $65.0 \%$, en mujeres $76.7 \%$ y $53.3 \%$ en hombres, lo que indicó que hubo 1.4 mujeres por cada hombre con SM. La mayoría de la muestra estudiada estaba en el rango de edad de 45 a 50 años con $27.3 \%$ mujeres y $34.7 \%$ hombres (Tabla 1). Nuestros resultados indican que hay diferencia significativa entre la frecuencia de SM entre hombres y mujeres de la población estudiada $(p$ $<.001$ ), esto confirma lo encontrado en otros estudios que presentan altas prevalencias del SM, mayormente en mujeres y en el área rural; evidenciándose que puede aumentar por diversos factores tales como la raza o etnia, malnutrición materno infantil, cambios en el estilo de vida (incluyendo el proceso de urbanización) y envejecimiento de la población (Bolado et al., 2015).

En general la prevalencia mundial de SM varía de $<10.0 \%$ a hasta $84.0 \%$, según la región, el entorno urbano o rural, la composición (sexo, edad, raza y etnia) de la población estudiada y la definición de SM utilizada (Desroches \& Lamarche, 2007; Kolovou, Anagnostopoulou, Salpea, \& Mikhailidis, 2007; Royer et al., 2007 ).

Es de importancia la detección precoz de las personas con SM, debido a la probabilidad incrementada de 3 veces de sufrir un infarto o accidente vascular cerebral, el doble de padecer ECV o morir por estos eventos y cinco veces incrementado el riesgo de padecer DM2 (International Diabetes Federation [IDF], 2006; Radu \& Stoica, 2016).

Con respecto a la asociación de SM en los diferentes grupos etarios, en que se dividió la muestra, se encontró que en hombres la edad está asociada en la década de los 40 y 60 años, $\left(\operatorname{chi}^{2}=9.26, p<.010\right)$. En tanto que, en las mujeres, la edad no está asociada específicamente a cada una de las décadas, la frecuencia de SM es similar en todas las décadas estudiadas ( $\left.\mathrm{chi}^{2}=0.4991, p=.779\right)$.); lo cual, se puede explicar por los cambios fisiológicos en la perimenopausia y postmenopausia, principalmente de tipo hormonal, que favorecen la acumulación de grasa visceral y disfunción del tejido adiposo. Un alto porcentaje de los participantes presentaron obesidad con una deposición excesiva de grasa corporal, particularmente en la región intraabdominal, que conduce a un aumento de ácidos grasos circulantes que promueven la resistencia a la insulina (RI) en el hígado, músculos y órganos principales que responden a las acciones metabólicas de la insulina (Lissón, 2017; Royer et al., 2007). En el estudio se evaluó la presencia de sobrepeso y obesidad mediante la medición del índice de masa corporal (IMC), se encontró en hombres y mujeres 50.0\% y 43.4\% con sobrepeso y $82.4 \%$ y $92.0 \%$ con obesidad, respectivamente, con SM.

Asimismo, los resultados evidencian que el $64.5 \%$ y el $78.1 \%$ de hombres y mujeres con SM respectivamente tenían familiares con DM2, siendo significativo únicamente en hombres $(p=.021)$.

En otros estudios realizados, se estableció la asociación entre DM2 y SM, debido a la presencia de resistencia a la insulina. Una de las consecuencias de la RI, es la disminución de adiponectina, que tiene como resultado el incremento de la producción hepática de glucosa (vía glucogenólisis y gluconeogénesis) y en células $\beta$ pancreáticas una inhibición disminuida de la concentración de glucagón, lo que también contribuye a la hiperglicemia (Lopes, Correa, Consolim, \& Egan, 2016). Si bien el SM y DM2 a menudo coexisten, los individuos con SM y sin DM2, tienen un riesgo significativo de desarrollarla (Punthakee, Goldenberg, \& Katz, 2018).

A los participantes con SM, se les evaluó el riesgo cardiovascular en los próximos 10 años, utilizando la estimación de Framingham. Los datos obtenidos indicaron un riesgo bajo en el $41.0 \%$ y $61.4 \%$ de hombres y mujeres respectivamente. Y un riesgo moderado en el $66.0 \%$ de hombres y $90.6 \%$ de mujeres. Se evidenció asociación significativa en hombres $(p=.0284)$ y en mujeres con el riesgo moderado $(p=.0030)$ y la presencia de SM. La importancia de evaluar el riesgo cardiovascular en la población de estudio está fundamentada en los hallazgos de varias investigaciones, que han indicado que la presencia de comorbilidades, y de factores asociados a un mayor riesgo, como la presencia de HTA, RI, anomalías lipídicas y obesidad, influyen significativamente a largo plazo en la morbilidad y mortalidad en pacientes sintomáticos y asintomáticos. Así 
como, que el SM presenta una estricta relación bidireccional con la Falla cardiaca, siendo responsable de un aumento constante de esta condición, con una prevalencia del $34.0 \%$ en la población general (Ervin, 2009; Redfield et al., 2003).

La triada de componentes de SM encontrada en el $73.0 \%$ de las mujeres participantes fue la hipertrigliceridemia, niveles séricos disminuidos de C-HDL y la circunferencia abdominal aumentada; y el $51.2 \%$ de los hombres presentó como tríada la hipertrigliceridemia, niveles séricos disminuidos de CHDL y la hiperglucemia en ayunas (Figura 1). Estas alteraciones metabólicas que presentan los individuos dependerán de las diferentes formas o grados de RI y del fenotipo de los componentes del SM que se presente en cada población (Radu \& Stoica, 2016).

La creciente prevalencia de obesidad y enfermedades crónicas, indican que los factores ambientales ejercen efectos a través de mecanismos epigenéticos. La alternancia y cambios en las marcas epigenéticas es probable que se deba a la dieta alta en grasas, las vías inflamatorias, el envejecimiento, y además, por trastornos neurológicos, estrés oxidativo, RI, obesidad, disfunción vascular y cánceres (Burdge et al., 2011; Simmons, 2011; Wang et al., 2012).

Los resultados relacionan los indicadores de la distribución de la grasa corporal, con situaciones de riesgo cardiovascular, así como la dislipidemia, hiperglucemia, presión arterial en hombres y mujeres adultos mayores. Por ello, se recomienda que la circunferencia de cintura y la presión arterial sencillas y fáciles de obtener, sean de uso rutinario en la evaluación de la obesidad y sus alteraciones, pues definitivamente permitirían a una mejor identificación de pacientes con alto riesgo de padecer enfermedades crónicas, como el Síndrome metabólico.

Estudios realizados en Guatemala, por Castañeda (2013) indican que el tratamiento del SM es multifactorial.

Es importante que, los participantes diagnosticados con SM en este estudio, comprendan la importancia que el ejercicio debe ser parte de la rutina diaria, así como la alimentación balanceada para lograr revertir el SM y evitar el desarrollo de otras comorbilidades, que deterioran su salud y estilo de vida con las correspondientes consecuencias económicas.

\section{Agradecimientos}

Al personal de consulta interna, externa y del laboratorio clínico del Hospital Nacional de San Marcos.

\section{ReFERENCIAS}

Alberti, K. G. M. M., \& Zimmet, P. (2005). The metabolic a new worldwide definition. The Lancet, 366(9491), 1059-1062. https://doi.org/10.1016/S0140-6736(05)67402-8

Alberti, K. G., Eckel, R. H., Grundy, S. M., Zimmet, P. Z., Cleeman, J. I., Donato, J. C. F., ... Smith, S. C. (2009). Harmonizing the metabolic syndrome: a joint interim statement of the international diabetes federation task force on epidemiology and prevention; National heart, lung, and blood institute. American heart association; World heart federation; International atherosclerosis society; and international association for the study of obesity. Circulation, 120(16), 1640-1645. https://doi.org/10.1161/CIRCULATIONAHA.109.192644

Bolado, V., Rivera, M., Soto, M., Blasco, G., \& Romero, L. (2015). Componentes clínicos del Síndrome Metabólico. Revista Médica de la Universidad Veracruzana, 2(15), 67-89.

Burdge, G. C., Hoile, S. P., Uller, T., Thomas, N. A., Gluckman, P. D., Hanson, M. A., \& Lillycrop, K. A. (2011). Progressive, transgenerational changes in offspring phenotype and epigenotype following nutritional transition. PloS one, 6(11). https://doi.org/10.1371/journal.pone.0028282

Castañeda, S. (2013). Tratamientos benéficos en disminución de riesgo cardiovascular en síndrome metabólico. Revista Guatemalteca de Cardiología, 23(1),21 - 28 
Cruz, E., Serrano, B., Jiménez, R., Villalobos, R., \& Murguía, M. (2015). Base de datos integral de tres encuestas nacionales de salud: Análisis del cambio de prevalencia de obesidad como ejemplo de uso. Revista de la Conferencia Cientifica Anual sobre Sindrome Metabólico, 1(2), 12-13.

Desroches, S., \& Lamarche, B. (2007). The evolving definitions and increasing prevalence of the metabolic syndrome. Applied Physiology, Nutrition, and Metabolism, 32(1), 23-32. https://doi.org/10.1139/h06-095

Escobedo, J., Schargrodsky, H., Champagne, B., Silva, H., Boissonnet, C., Vinueza, R., ... Wilson, E. (2009). Prevalence of the Metabolic Syndrome in Latin America and its association with sub-clinical carotid atherosclerosis: the CARMELA cross sectional study. Cardiovascular Diabetology, 8(52), 1-9. https://doi.org/10.1186/1475-2840-8-52

Ervin, R. B. (2009). Prevalence of metabolic syndrome among adults 20 years of age and over, by sex, age, race and ethnicity, and body mass index: United States, 2003-2006. National Health Statistics Reports, 13, 1-7.

Grundy, S. M., Cleeman, J. I., Daniels, S. R., Donato, K. A., Eckel, R. H., Franklin, B. A., ... \& Costa, F. (2005). Diagnosis and management of the metabolic syndrome: an American Heart Association/National Heart, Lung, and Blood Institute scientific statement. Circulation, 112(17), 2735-2752.

https://doi.org/10.1161/CIRCULATIONAHA.105.169404

Huang, P. L. (2009). A comprehensive definition for metabolic syndrome. Disease Models \& Mechanisms, 2(5-6), 231-237. https://doi.org/10.1242/dmm.001180

International Diabetes Federation (2006). The IDF consensus worldwide definition of the Metabolic Syndrome. Recuperado de https://www.pitt.edu/ super1/Metabolic/IDF1.pdf

Kee, C.C., Sumarni, M.G., Lim, K. H., Selvarajah, S., Hanniff, J., Helen, G., ... Amal, N. (2017). Association of BMI with risk of CVD mortality and all-cause mortality. Public Health Nutrition, 20(7), 1226-1234. https://doi.org/10.1017/S136898001600344X

Kolovou, G. D., Anagnostopoulou, K. K., Salpea, K. D., \& Mikhailidis, D. P. (2007). The prevalence of metabolic syndrome in various populations. The American Journal of the Medical Sciences, 333(6), 362-371. https://doi.org/10.1097/MAJ.0b013e318065c3a1

Lahsen, R. (2014). Síndrome metabólico y diabetes. Revista Médica Clínica Condes, 25(1), 47-52. https://doi.org/10.1016/S0716-8640(14)70010-0

Lissón, R. (2017). Impacto de la obesidad en la salud de la mujer adulta mayor. Revista Peruana de Ginecología y Obstetricia, 63(4), 615-621. https://doi.org/10.31403/rpgo.v63i2037

Lopes, H. F., Corrêa-Giannella, M. L., Consolim-Colombo, F. M., \& Egan, B. M. (2016). Visceral adiposity syndrome. Diabetology \& metabolic syndrome, 8(1), 1-8. https://doi.org/10.1186/s13098-016-0156-2

López, P., Sánchez, R., Díaz, M., Cobos, L., Bryce, A., Parra, J., ... Zanchetti, A. (2013). Consenso latinoamericano de hipertensión en pacientes con diabetes tipo 2 y síndrome metabólico. Acta Médica Colombiana, 38(3), 154-172.

McKenney, R. L., \& Short, D. K. (2011). Tipping the balance: the pathophysiology of obesity and type 2 diabetes mellitus. The Surgical clinics of North America, 91(6), 1139-1148. https://doi.org/10.1016/j.suc.2011.08.007

Ministerio de Salud Pública y Asistencia Social (2018). Análisis de Situación: Enfermedades No Transmisibles en Guatemala. Recuperado de http://epidemiologia.mspas.gob.gt/files/Publicaciones\%202018/Enfermedades \%20No\%20Transmisibles/Analisis\%20anual\%20ENT\%202018.pdf

National Cholesterol Education Program. (2001). Executive summary of the third report of the National Cholesterol Education Program expert panel on detection, evaluation, and treatment of high blood cholesterol in adults. Journal of the American Medical Association, 285(19), 2486-2497. https://doi.org/10.1001/jama.285.19.2486

Ninatanta, J., Núñez, L., García, S., \& Romaní, F. (2016). Frecuencia de síndrome metabólico en residentes de una región andina del Perú. Revista Peruana de Medicina Experimental y Salud Pública, 33(4), 640-650. https://doi.org/10.17843/rpmesp.2016.334.2546

Olijhoek, J. K., Van Der Graaf, Y., Banga, J. D., Algra, A., Rabelink, T. J., \& Visseren, F. L. J. (2004). The Metabolic Syndrome is associated with advanced vascular damage in patients with coronary heart disease, stroke, peripheral arterial disease or abdominal aortic aneurysm. European Heart Journal, 25(4), 342-348. https://doi.org/10.1016/j.ehj.2003.12.007 
Punthakee, Z., Goldenberg, R., \& Katz, P. (2018). Diabetes Canada Clinical Practice Guidelines Expert Committee Definition, Classification and Diagnosis of Diabetes, Prediabetes and Metabolic Syndrome. Canadian Journal of Diabetes, 42, S10-S15. https://doi.org/10.1016/j.jcjd.2017.10.003

Radu, C., \& Stoica, V. (2016). Metabolic syndrome cardiovascular and metabolic, complex, difficult to quantify risk factor. Modern Medicine, 23(1), 54-59.

Redfield, M. M., Jacobsen, S. J., Burnett, J. C., Mahoney, D. W., Bailey, K. R., \& Rodeheffer, R. J. (2003). Burden of systolic and diastolic ventricular dysfunction in the community: appreciating the scope of the heart failure epidemic. JAMA, 289(2), 194-202. https://doi.org/10.1001/jama.289.2.194

Royer, M., Castelo-Branco, C., Blümel, J. E., Chedraui, P. A., Danckers, L., \& Bencosme, A. (2007). Collaborative Group for Research of the Climacteric in Latin America The US national cholesterol education programme adult treatment panel III (NCEP ATP III): prevalence of the metabolic syndrome in postmenopausal Latin American women. Climacteric, 10, 164-170. https://doi.org/10.1080/13697130701258895

Simmons, R. (2011). Epigenetics and maternal nutrition: Nature v. nurture. Proceedings of the Nutrition Society, 70(1), 73-81. https://doi.org/10.1017/S0029665110003988

Tsukiyama, H., Nagai, Y., Matsubara, F., Shimizu, H., Iwamoto, T., Yamanouchi, E., ...Tanaka, Y. (2016). Proposed cut-off values of the waist circumference for metabolic syndrome based on visceral fat volume in a Japanese population. Journal of Diabetes Investigation, 7(4), 587-593. https://doi.org/10.1111/jdi.12454

Tzorin, P., Castellanos, A., Chopox, L., Arenas, A., Gaitán, C., Ramírez, M., \& Ortiz, D. (2015). Frecuencia de Síndrome Metabólico en personal de mantenimiento que labora dentro del campus central de la Universidad de San Carlos de Guatemala, en el año 2014. Revista Cientifica de la Facultad de Ciencias Químicas y Farmacias, 25(1), 9-17.

Villalta, M. (2016). Prevalencia de síndrome metabólico en mujeres indígenas mayores de 45 años. Revista Médica de la Universidad Rafael Landivar, Facultad de Ciencias de la Salud, Guatemala. 155(1), 33-36. https://doi.org/10.36109/rmg.v155i1.31

Wang, J., Wu, Z., Li, D., Li, N., Dindot, S. V., Satterfield, M. C., ...Wu, G. (2012). Nutrition, epigenetics, and metabolic syndrome. Antioxidants \& Redox Signaling, 17(2), 282-301. https://doi.org/10.1089/ars.2011.4381

Wilson, P. W., D’Agostino, R. B., Parise, H., Sullivan, L., \& Meigs, J. B., (2005). Metabolic syndrome as a precursor of cardiovascular disease and type 2 diabetes mellitus. Circulation, 112(20), 3066-3072. https://doi.org/10.1161/CIRCULATIONAHA.105.539528

Wong, R. (2012). Prevalencia de sindrome metabólico y factores asociados en cinco ciudades principales de países centro americanos, 2003-2007. (Tesis de maestría), Universidad del Valle de Guatemala, Guatemala.

\title{
Copyright (c) 2021 Marta Julia Saquic, Deris Roselvy Velásquez y Alba Marina Valdés de Garáa
}

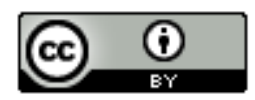

Este texto está protegido por una licencia $\underline{\text { Creative Commons }} \underline{40}$

\begin{abstract}
Usted es libre para Compartir — copiar y redistribuir el material en cualquier medio o formato- y Adaptar el documento — remezclar, transformar y crear a partir del material- para cualquier propósito, , incluso para fines comerciales, siempre que cumpla la condición de:

Atribución: Usted debe dar crédito a la obra original de manera adecuada, proporcionar un enlace a la licencia, e indicar si se han realizado cambios. Puede hacerlo en cualquier forma razonable, pero no de forma tal que sugiera que tiene el apoyo del licenciante o lo recibe por el uso que hace de la obra.
\end{abstract}

Resumen delicencia - Textocompletodela licencia 